\title{
ON A SET OF NORMAL SUBGROUPS
}

\author{
by I. D. MACDONALD
}

(Received 4 April, 1961)

1. The commutator $[a, b]$ of two elements $a$ and $b$ in a group $G$ satisfies the identity

$$
a b=b a[a, b] .
$$

The subgroups we study are contained in the commutator subgroup $G^{\prime}$, which is the subgroup generated by all the commutators.

The group $G$ is covered by a well-known set of normal subgroups, namely the normal closures $\{g\}^{G}$ of the cyclic subgroups $\{g\}$ in $G$. In a similar way one may associate a subgroup $K(g)$ with each element $g$, by defining $K(g)$ to be the subgroup generated by the commutators $[g, x]$ as $x$ takes all values in $G$. These subgroups generate $G^{\prime}$ (but do not cover $G^{\prime}$ in general), and are normal in $G$ in consequence of the identical relation

$$
[g, x]^{y}=[g, y]^{-1}[g, x y]
$$

holding for all $g, x$ and $y$ in $G$. (By $u^{b}$ we mean $b^{-1} a b$.) It is easy to see that

$$
\{g\}^{G}=\{g, K(g)\} .
$$

The subgroups $K(g)$ appear in a number of situations. For instance, it is shown in Theorem 3 of [1] that if every $K(g)$ in $G$ is abelian, then the commutator subgroup $G^{\prime \prime}$ of $G^{\prime}$ lies in the centre of $G$ and has exponent 2. Again, every $K(g)$ is finite if and only if every element of $G$ has just a finite number of conjugates. One part of this statement is clear, and to prove the other part suppose that every element of $G$ has only a finite number of conjugates. Then any subgroup $K(g)$ is generated by a finite set of commutators of the form $[g, x]$ for certain elements $x$; each $[g, x]$ has finite order by Theorem 5.1 in [2]. These facts, and the condition on conjugates in $G$, and use of Corollary 5.21 of [2], show that each $K(g)$ is finite. We further note that, because of Theorem 3.1 of [3], each $K(g)$ is boundedly finite if and only if $G^{\prime}$ is finite.

In $\S 2$ we consider groups $G$ in which each $K(g)$ contains elements of the form $[g, x]$ only. This with minimal condition on the $K(g)$ appears to be a strong restriction on $G$, which will be shown to be a $Z A$ group. An unusual feature of this result is that conditions on $G^{\prime}$ give a conclusion on the structure of $G$, not just of $G^{\prime}$. In $\S 3$ we turn to groups $G$ in which each $K(g)$ is cyclic. As it can be shown that $G^{\prime}$ is then locally cyclic, it is worth considering groups with each $K(g)$ locally cyclic. We show that again $G^{\prime}$ is locally cyclic.

2. The subgroup $K(g)$ contains only commutators of the form $[g, x]$ if and only if the equations

$$
\begin{aligned}
& {[g, x][g, y]=\left[g, z_{1}\right],} \\
& {[g, x]^{-1}=\left[g, z_{2}\right]}
\end{aligned}
$$


can be solved for $z_{1}$ and $z_{2}$, the elements $x$ and $y$ being arbitrary. An equivalent condition is, clearly, that

$$
[g, y]^{-1}[g, x y]=[g, z]
$$

should be soluble for $z$. By (A) and (D) another equivalent condition is that

$$
[g, x]^{y}=[g, z]
$$

should be soluble for $z$.

A further condition may be obtained when it is noted that solubility of (B) is sufficient for solubility of (C). For if there is an element $z_{0}$ such that

$$
\left[g, x^{-1}\right]\left[g, x^{-1}\right]=\left[g, z_{0}\right]
$$

then we have successively

$$
\begin{aligned}
g^{-1} x g x^{-1} g^{-1} x g x^{-1} & =g^{-1} z_{0}^{-1} g z_{0}, \\
x^{-1} g^{-1} x g & =g^{-1} x^{-1} z_{0}^{-1} g z_{0} x, \\
{[g, x]^{-1} } & =\left[g, z_{0} x\right] ;
\end{aligned}
$$

thus $z_{2}=z_{0} x$ is a solution of (C).

Next we suppose that every $K(g)$ in $G$ satisfies such a condition, and in addition we impose a minimal condition.

THEOREM 1. Let each subgroup $K(g)$ of the group $G$ consist of commutators of the form $[g, x]$, and let $G$ be such that the minimal condition holds for the subgroups $K(g)$. Then $a$ non-trivial element of each subgroup $\{g\}^{G}$ lies in the centre of $G$, provided that $g \neq 1$.

Proof. If $g$ is an arbitrary element of $G$ we may suppose that $K(g)$ is not the trivial subgroup 1, for otherwise $g$ is in the centre of $G$ and the theorem holds. In $K(g)$ we choose a minimal non-trivial subgroup of the form $K\left(g_{0}\right)$-this exists because of the minimal condition-and we choose an element $h \neq 1$ in $K\left(g_{0}\right)$. As we have $K(h) \subseteq K\left(g_{0}\right)$, we see that $K(h)=K\left(g_{0}\right)$ or $K(h)=1$. In the former case $h^{-1} \in K\left(g_{0}\right)=K(h)$, so by hypothesis there is an element $x$ in $G$ for which

$$
h^{-1}=[h, x]=h^{-1} x^{-1} h x,
$$

implying that $h=1$, a contradiction. Therefore we must have $K(h)=1$, that is, $h$ is central in $G$. As $h \in K\left(g_{0}\right) \subseteq K(g) \subseteq\{g\}^{G}$, the theorem follows.

COROLLARY 1. Under the hypotheses of Theorem $1, G$ is a ZA group.

Proof. By a $Z A$ group is meant a group with an ascending central series which eventually exhausts the group. As Theorem 1 shows that $G$ has a non-trivial centre, the corollary follows once it is verified that the properties required in Theorem 1 persist in homomorphic images of $G$. This is elementary.

COROLlaRY 2. A group in which each $K(g)$ consists of elements $[g, x]$, and in which every element has only a finite number of conjugates, is $Z A$. 
Proof. We remarked earlier that this finiteness condition on conjugates is equivalent to each $K(g)$ being finite. Application of Corollary 1 completes the proof.

In particular, finite groups with the condition of Theorem 1 on the $K(g)$ are nilpotent. However, it is not difficult to see that the class of nilpotency is arbitrary.

We are in a position to show that neither of the following conditions on a group $G$ implies the other:

(i) $G^{\prime}$ consists of commutators;

(ii) for each $g$ in $G, K(g)$ consists of the commutators $[g, x]$ as $x$ varies in $G$.

Though many finite non-nilpotent groups satisfy (i), no such group satisfies (ii), by a remark above. For examples, we refer to Ore's paper [4], where it is established that the alternating groups of finite degree greater than or equal to 5 satisfy (i). On the other hand, it is clear that any group that is nilpotent of class 2 satisfies (ii), and it seems to be well-known that such a group need not satisfy (i). We present a supporting example as no record of one can be readily found.

The example $G_{1}$ is simply the free nilpotent group of class 2 on 4 generators $a_{1}, a_{2}, a_{3}, a_{4}$; if $c_{i j}=\left[a_{i}, a_{j}\right]$ for $1 \leqq i<j \leqq 4$, the relations in $G_{1}$ are

$$
\left[c_{i j}, a_{k}\right]=1
$$

for $1 \leqq i<j \leqq 4$ and $1 \leqq k \leqq 4$, and their consequences. Each element of $G_{1}$ has a unique representation in the form

$$
a_{1}^{\alpha_{1}} a_{2}^{\alpha_{2}} a_{3}^{\alpha_{3}} a_{4}^{\alpha_{4}} \prod c_{i j}^{\alpha_{j}}
$$

where the product is taken over all $i$ and $j$ with $1 \leqq i<j \leqq 4$. So an arbitrary commutator may be written as

$$
\left[a_{1}^{\alpha_{1}} a_{2}^{\alpha_{2}} a_{3}^{\alpha_{3}} a_{4}^{\alpha_{4}}, \quad a_{1}^{\beta_{1}} a_{2}^{\beta_{2}} a_{3}^{\beta_{3}} a_{4}^{\beta_{4}}\right],
$$

which may be simplified by use of the defining relations to

$$
\prod_{1 \leqq i<j \leqq 4} c_{i j}^{\delta_{i j} j} \text {, }
$$

where $\delta_{i j}=\alpha_{i} \beta_{j}-\alpha_{j} \beta_{i}$. It may be verified directly that the $\delta_{i j}$ satisfy

$$
\delta_{12} \delta_{34}-\delta_{13} \delta_{24}+\delta_{14} \delta_{23}=0 .
$$

If $c_{13} c_{24}$ is the commutator of two elements of $G_{1}$, the uniqueness of the representation shows that we must have $\delta_{12}=\delta_{34}=\delta_{14}=\delta_{23}=0, \delta_{13}=\delta_{24}=1$. Since these $\delta_{i j}$ do not satisfy the above identity, we have a contradiction; so $c_{13} c_{24}$ is not a commutator, and $G_{1}$ satisfies (ii) but not (i). We note without proof that finite groups with similar properties may be found by taking factor groups of $G_{1}$.

We now construct a group $G_{2}$ with the purpose of showing that the minimal condition cannot be omitted from the hypotheses of Theorem 1 and its corollaries. Let $U$ be a multiplicative group isomorphic to the additive group of rationals, with $u$ in $U$ corresponding to the rational 1; thus $u^{r}$ corresponds to the rational $r$, and

$$
u^{r_{1}} u^{r_{2}}=u^{r_{1}+r_{2}}
$$


for any rationals $r_{1}$ and $r_{2}$. The unit element of $U$ is $u^{0}$, which will be written as 1 . Now $U$ has an automorphism $\alpha_{0}$ such that $x \alpha_{0}=x^{-1}$ for all $x \in U$, and automorphisms $\alpha_{n}$ such that $x \alpha_{n}=x^{p_{n}}$ for all $x \in U$, where $p_{n}$ is the $n$th odd prime. These automorphisms $\alpha_{0}, \alpha_{1}, \alpha_{2}, \ldots$ generate an abelian group $A$ of automorphisms of $U$. The example $G_{2}$ is the splitting extension of $U$ by $A$, and we suppose that the element $a_{i}$ of $G_{2}$ corresponds to the automorphism $\alpha_{i}$ of $A$.

The proof that $K(g)$ consists of elements $[g, x]$ is in two parts.

(i) Let $[g, u] \neq 1$, say $u^{g}=u^{\rho}$ for some rational $\rho \neq 1$. Clearly $K(g) \subseteq U$, and we can in fact solve the equation

$$
[g, x]=u^{\sigma}
$$

for $x$, where $\sigma$ is any rational. It is easy to verify that $x=u^{\sigma /(1-\rho)}$ is a solution. Thus $K(g)$ has the required property.

(ii) Let $[g, u]=1$. We may assume that $g$ is not central in $G$, for then $K(g)=1$ and the result is trivial. Thus $g=a u^{\tau}$, where $a$ is central in $G$ and $\tau \neq 0$, and $K(g)$ is generated by elements of the form $u^{\phi \tau}$, where $\phi$ is rational with even numerator and odd denominator. Consequently all elements of $K(g)$ have the same form as $u^{\phi \tau}$, and we have to solve an equation of the form

or equivalently

$$
[g, x]=u^{\phi \varepsilon},
$$

$$
\left(u^{r}\right)^{x}=u^{(\phi+1) r},
$$

for $x$. The form of $\phi$ shows that $\phi+1$ is the quotient of two odd integers and, in particular, that $\phi+1$ is non-zero:

$$
\phi+1=(-1)^{e} p_{i_{1}}^{\varepsilon_{1}} p_{i_{2}}^{\ell_{2}} \ldots p_{i_{s}}^{\ell_{s}},
$$

where $\varepsilon=0$ or 1 , the $\varepsilon_{1}$ are non-zero integers, and $s \geqq 0$. Then for $x$ we take the element $a_{0}^{\varepsilon} a_{i_{1}}^{z_{1}} a_{i_{2}}^{\varepsilon_{2}} \ldots a_{i_{s}}^{\varepsilon_{s}}$

In either case $K(g)$ contains no elements other than the $[g, x]$. But $G_{2}$ is not a $Z A$ group as its subgroup $\left\{u, a_{0}\right\}$ is certainly not $Z A$.

3. In this section we discuss groups with each $K(g)$ cyclic, after a digression on similar conditions for $\{g\}^{G}$.

More precisely, we start by proving the equivalence of the three following conditions on the group $G$ :

(i) every subgroup is normal;

(ii) every $\{g\}^{G}$ is cyclic;

(iii) every $\{g\}^{G}$ is locally cyclic.

Clearly (i) implies (ii) and (ii) implies (iii), leaving us to show that (iii) implies (i). Consider the subgroup $\left\{g, g^{x}\right\}$, where $x$ is an arbitrary element of the group $G$, which satisfies (iii). As $\left\{g, g^{x}\right\}$ is cyclic, being a finitely generated subgroup of $\{g\}^{G}$, we have

$$
g=h^{\alpha}, \quad g^{x}=h^{\beta}
$$

for some $h$ and some coprime $\alpha$ and $\beta$; thus

$$
\left(h^{\alpha}\right)^{x}=h^{\beta}, \quad x^{h^{\alpha}}=x h^{\alpha-\beta} .
$$


Now $\left\{x, x h^{\alpha-\beta}\right\}$ is cyclic and so abelian. Hence we have

$$
h^{a(\alpha-\beta)}=\left(h^{\alpha(\alpha-\beta)}\right)^{x}=h^{\beta(\alpha-\beta)}, \quad h^{(\alpha-\beta)^{2}}=1 .
$$

If $h$ has infinite order, then $\alpha=\beta$, that is $[g, x]=1$. If $h$ has finite order, then the numbers $\alpha, \beta$ and $(\alpha-\beta)^{2}$ are coprime in pairs; so $g^{x}$ lies in $\{g\}$. In either case $\{g\}$ is normal in $G$, which at once gives (i).

The theorem of Dedekind and Zassenhaus describes completely the groups satisfying (i); see [5, pp. 159-161]. Such a group, if non-abelian, is the direct product of a quaternion group, an abelian group of exponent two, and an abelian group with every element of odd order.

When we impose the condition that every $K(g)$ is cyclic or locally cyclic (see Theorems 2 and 3), we cannot hope to determine more than the structure of $G^{\prime}$.

In preparation for both these theorems we prove now that $G^{\prime}$ is abelian when every $K(g)$ in $G$ is locally cyclic. If $c=[a, b]$ and $d=\left[a^{\prime}, b^{\prime}\right]$ are arbitrary commutators in $G$, then the subgroup

$$
\left\{d, d^{a^{-1}}, d^{a^{-1} b^{-1}}, d^{a^{-1} b^{-1} a}, d^{[a, b]}\right\}
$$

of $K\left(a^{\prime}\right)$ is cyclic, with generator $h$ say. Hence we have

$$
d=h^{\alpha}, \quad d^{a-1}=h^{\beta}, \quad d^{a^{-1} b-1}=h^{\gamma}, \quad d^{a^{-1} b^{-1} a}=h^{\delta}, \quad d^{[a, b]}=h^{e} .
$$

It follows that

$$
h^{\alpha \gamma}=d^{\gamma}=\left(h^{\beta \gamma}\right)^{a}=\left(d^{a-1 b-1 a}\right)^{\beta}=h^{\beta \delta}=\left(d^{a-1}\right)^{\delta}=\left(h^{\gamma \delta}\right)^{b}=\left(d^{[a, b]}\right)^{\gamma}=h^{e \gamma}, \quad h^{\gamma(\alpha-\varepsilon)}=1 .
$$

Successive transformations by $b, b a, a$ and $a b$ give

Since

$$
h^{\beta(\alpha-\varepsilon)}=h^{\alpha(\alpha-\varepsilon)}=h^{\delta(\alpha-\varepsilon)}=h^{\varepsilon(\alpha-\varepsilon)}=1 .
$$

we have

$$
h \in\left\{h^{\alpha}, h^{\beta}, h^{\gamma}, h^{\delta}, h^{e}\right\},
$$

$$
h^{\alpha-\varepsilon}=1 \text {, }
$$

that is, $d=d^{[a, b]}$. Therefore, as arbitrary commutators $c$ and $d$ in $G$ commute, we conclude that $G^{\prime}$ is abelian.

We state once and for all the fact that if every $K(g)$ in $G$ is locally cyclic, or cyclic, then the same property is to be found in all subgroups and factor groups of $G$.

It is convenient to consider first the case in which $G^{\prime}$ is finite.

THEOREM 2. Let $G$ be a group with finite commutator subgroup. Then $G^{\prime}$ is cyclic if and only if $K(g)$ is cyclic for each $g$ in $G$.

Proof. When every $K(g)$ is cyclic, we use induction on the order of $G^{\prime}$ to establish that $G^{\prime}$ is cyclic. Suppose that the abelian group $G^{\prime}$ has two distinct non-trivial Sylow subgroups $S_{p}$ and $S_{q}$. Each of these is characteristic in $G^{\prime}$ and so normal in $G$. By the induction hypothesis, $G / S_{p}$ has its commutator subgroup $G^{\prime} \mid S_{p}$ cyclic, and similarly $G^{\prime} / S_{q}$ is cyclic. Therefore $G^{\prime}$ is cyclic.

Next suppose that $G^{\prime}$ is a non-trivial $p$-group for some prime $p$. In this case $G^{\prime}$ contains a 
subgroup $N$, of order $p$, which is normal in $G$, because we may take $N$ to be the subgroup of order $p$ in any non-trivial $K(g)$. We have $G^{\prime} \mid N$ cyclic. If $G^{\prime}$ is non-cyclic, then it is the direct product of cyclic subgroups of orders $p$ and $p^{n}$ respectively, where $n \geqq 1$, and we show that this case is impossible.

We must have $n=1$; for if $n>1$, and if $H$ denotes the subgroup of $G^{\prime}$ generated by the pth powers of all its elements, then $H \supset 1$ and $G^{\prime} / H$ is cyclic by the induction hypothesis, which is impossible. Therefore $G^{\prime}$ is the direct product of subgroups $K\left(a_{1}\right)$ and $K\left(a_{3}\right)$, with

say. Clearly

$$
\left[a_{1}, a_{2}\right]=c_{12} \neq 1, \quad\left[a_{3}, a_{4}\right]=c_{34} \neq 1,
$$

$$
\begin{gathered}
K\left(a_{1}\right)=K\left(a_{2}\right)=\left\{c_{12}\right\}, \quad K\left(a_{3}\right)=K\left(a_{4}\right)=\left\{c_{34}\right\}, \\
{\left[a_{2}, a_{3}\right] \in K\left(a_{2}\right) \cap K\left(a_{3}\right)=1, \quad\left[a_{1}, a_{4}\right] \in K\left(a_{1}\right) \cap K\left(a_{4}\right)=1 .}
\end{gathered}
$$

Consider $K\left(a_{1} a_{3}\right)$. We have

$$
\left(a_{1} a_{3}\right)^{a_{2}}=a_{1} c_{12} a_{3}=a_{1} a_{3} c_{12}^{a_{3}}, \quad\left(a_{1} a_{3}\right)^{a_{4}}=a_{1} a_{3} c_{34},
$$

and therefore

$$
K\left(a_{1} a_{3}\right) \supseteq\left\{c_{12}, c_{34}\right\} .
$$

This contradicts the fact that $K\left(a_{1} a_{3}\right)$ is cyclic, and completes the proof of Theorem 2.

A lemma of a technical nature precedes Theorem 3.

Lemma. A finitely generated group $\left\{c_{1}, c_{2}, \ldots, c_{n}\right\}$ is cyclic if and only if $\left\{c_{i}, c_{j}\right\}$ is cyclic for all $i$ and $j$ with $1 \leqq i<j \leqq n$.

Proof. We establish the less trivial part of the lemma in several stages. If each $\left\{c_{i}, c_{j}\right\}$ is cyclic, then $\left[c_{i}, c_{j}\right]=1$ and the group $A$ generated by $c_{1}, c_{2}, \ldots, c_{n}$ is abelian. Suppose that $A$ is a $p$-group. The fact that $\left\{c_{i}, c_{j}\right\}$ is a cyclic $p$-group shows that $c_{i} \in\left\{c_{j}\right\}$ or $c_{j} \in\left\{c_{i}\right\}$; so either $c_{i}$ or $c_{j}$ can be omitted from the given system of generators. An obvious induction on $n$ shows that $A$ is cyclic.

Suppose next that $A$ is periodic. For an arbitrary prime $p$ we choose elements $c_{i p}$ which generate the Sylow $p$-subgroup of $\left\{c_{i}\right\}$, for $1 \leqq i \leqq n$. The generators $c_{1 p}, c_{2 p}, \ldots, c_{n p}$ of the Sylow p-subgroup of $A$ inherit the property of the generators of $A$; therefore each Sylow $p$-subgroup of $A$ is cyclic. It follows that $A$ is cyclic.

There remains the case in which $A$ is infinite, though the periodic subgroup of $A$ is finite as $A$ is finitely generated. If this subgroup has order $m$, we consider the group $A / M$, where $M$ is generated by the $m$ th powers of the elements of $A$. Now $A / M$ is finite, and its generators $c_{1} M, c_{2} M, \ldots, c_{n} M$ inherit the property of the generators of $A$; so $A / M$ must be cyclic. Its order is at most $m$. As $A$ has elements of infinite order and its periodic part has order $m$, we must have $m=1$.

Therefore we consider the factor group $A / S$ of the torsion-free group $A$, where $S$ is generated by all squares in $A$. It is finite, and so cyclic. But $A$, having the same number of generators as $A / S$, is then cyclic.

This completes the proof of the lemma. 
THEOREM 3. The commutator subgroup of the group $G$ is locally cyclic if and only if $K(g)$ is locally cyclic for each $g$ in $G$.

Proof. It is enough to show that if each $K(g)$ is locally cyclic, then so is $G^{\prime}$, which was shown above to be abelian. That is, we wish to show that any finite set of elements of $G^{\prime}$ generates a cyclic subgroup, or (equivalently) that any finite set of commutators generates a cyclic subgroup. The lemma reduces this problem to that of showing that any pair of commutators generates a cyclic subgroup.

Let $\left[a_{1}, a_{2}\right]$ and $\left[a_{3}, a_{4}\right]$ be any two commutators. As we work in the subgroup $\left\{a_{1}, a_{2}, a_{3}, a_{4}\right\}$ only, we shall take this to be $G$. If $c_{i j}=\left[a_{i}, a_{j}\right]$ for $1 \leqq i \leqq 4$ and $1 \leqq j \leqq 4$, our aim is to show that $\left\{c_{12}, c_{34}\right\}$ is cyclic. We suppose that $c_{12} \neq 1$ and $c_{34} \neq 1$.

First we discuss the case when $c_{13}=c_{24}=1$. Then

$$
\left(a_{1} a_{4}\right)^{a_{2}}=a_{1} c_{12} a_{4}=a_{1} a_{4} c_{12}^{a_{4}}, \quad\left(a_{1} a_{4}\right)^{a_{3}}=a_{1} a_{4} c_{34}^{-1}
$$

as $c_{12}$ and $c_{34}$ therefore lie in the locally cyclic group $K\left(a_{1} a_{4}\right)$, we see that $\left\{c_{12}, c_{34}\right\}$ is cyclic. We may, and shall, assume from here onwards that $c_{13} \neq 1$.

Next, suppose that $c_{12}$ has finite order. Then $c_{13}$ also has finite order, as $\left\{c_{12}, c_{13}\right\}$ is a cyclic subgroup of $K\left(a_{1}\right)$. Similarly $c_{34}$ has finite order, and indeed each subgroup $K\left(a_{i}\right)$ for $1 \leqq i \leqq 4$ is periodic, as it contains a non-trivial element of finite order. Now an arbitrary commutator $c$ in $G$ may be written as

$$
\left[x_{1} x_{2} \ldots x_{r}, y_{1} y_{2} \ldots y_{s}\right]
$$

where each $x_{i}$ and each $y_{i}$ is one of $a_{i}^{ \pm 1}$ for $1 \leqq i \leqq 4$, and the well-known identical relations

$$
[x y, z]=[x, z]^{y}[y, z], \quad\left[x^{-1}, y\right]=[y, x]^{x^{-1}}
$$

may be used to expand $c$ as the product of certain conjugates of the commutators $c_{i j}$. This proves that $G^{\prime} \subseteq K\left(a_{1}\right) K\left(a_{2}\right) K\left(a_{3}\right)$; hence $G^{\prime}$ is periodic. Because each $c_{i j}$ has finite order it generates a characteristic subgroup of $K\left(a_{i}\right)$, and so a normal subgroup of $G$. It follows that $c_{i j}$ has a finite number of conjugates. As $G^{\prime}$ is finitely generated and periodic, $G^{\prime}$ is finite. By Theorem $2, G^{\prime}$ is cyclic, and so is its subgroup $\left\{c_{12}, c_{34}\right\}$. We shall, therefore, in future suppose that $c_{12}$ has infinite order, which clearly implies that $c_{34}$ has infinite order.

In this case we investigate the structure of $G^{\prime}$ and its embedding in $G$. If $d$ is a generator of the cyclic subgroup $\left\{c_{12}, c_{13}\right\}$ of $K\left(a_{1}\right)$, we have

$$
c_{12}=d^{\alpha}, \quad c_{13}=d^{\beta}
$$

for some $\alpha \neq 0$ and $\beta \neq 0$; so $c_{12}^{\beta}=c_{13}^{\alpha}$. Similar consideration of $K\left(a_{3}\right)$ gives $c_{13}^{\beta^{\prime}}=c_{34}^{\alpha^{\prime}}$ for some $\alpha^{\prime} \neq 0$ and some $\beta^{\prime} \neq 0$. These results combine to give

$$
c_{12}^{\gamma_{12}}=c_{34}^{\gamma_{34}}
$$

where $\gamma_{12}=\beta \beta^{\prime} \neq 0, \gamma_{34}=\alpha \alpha^{\prime} \neq 0$. It is easy to see that a relation of the same sort holds when $c_{34}$ is replaced by any non-trivial commutator among $c_{13}, c_{14}, c_{23}, c_{24}$, because $K\left(a_{1}\right)$ and $K\left(a_{2}\right)$ are locally cyclic. 
Now let $x$ be an arbitrary element of $G$, and let $d_{12}$ be a generator of the cyclic subgroup $\left\{c_{12}, c_{12}^{x}\right\}$ of $K\left(a_{1}\right)$, so that

$$
c_{12}=d_{12}^{\lambda}, \quad c_{12}^{x}=d_{12}^{\mu},
$$

where $\lambda$ and $\mu$ are coprime; thus

$$
\left(d_{12}^{\lambda}\right)^{x}=d_{12}^{\mu} .
$$

On raising both sides to the power $\gamma_{12}$ and using (*), we find that

$$
\left(c_{34}^{\lambda y_{34}}\right)^{x}=c_{34}^{\mu \gamma_{34}} \text {. }
$$

Let $d_{34}$ generate the cyclic subgroup $\left\{c_{34}, c_{34}^{x}\right\}$, so that

$$
c_{34}=d_{34}^{\theta}, \quad c_{34}^{x}=d_{34}^{\omega}, \quad\left(d_{34}^{\theta}\right)^{x}=d_{34}^{\omega},
$$

where $\theta$ and $\omega$ are coprime. This last relation gives

We therefore have

$$
\left(c_{34}^{\theta \gamma_{34}}\right)^{x}=c_{34}^{\omega \gamma_{34}} .
$$

$$
c_{34}^{\mu \theta \gamma_{34}}=\left(c_{34}^{\lambda \theta \gamma_{34}}\right)^{x}=c_{34}^{\lambda \omega \gamma_{34}}, \quad c_{34}^{(\mu \theta-\lambda \omega) \gamma_{34}}=1 .
$$

It follows, since $c_{34}$ has infinite order, that

$$
\mu \theta-\lambda \omega=0 ;
$$

and because $\lambda$ and $\mu$ are coprime, and $\theta$ and $\omega$ are coprime, we have $\lambda=\theta, \mu=\omega$.

Therefore we have

$$
c_{34}=d_{34}^{\lambda}, \quad c_{34}^{x}=d_{34}^{\mu},
$$

where $x, \lambda$ and $\mu$ have the meanings explained above. A similar argument will show that

$$
c_{i j}=d_{i j}^{\lambda}, \quad c_{i j}^{x}=d_{i j}^{\mu}
$$

for a suitable element $d_{i j}$, where $1 \leqq i<j \leqq 4$. When $x=a_{k}$, we shall write $\lambda$ and $\mu$ as $\lambda_{k}$ and $\mu_{k}$ respectively, for $1 \leqq k \leqq 4$.

There is one case in which (*) at once shows that $\left\{c_{12}, c_{34}\right\}$ is cyclic, namely when this subgroup is torsion-free. If $\left|\gamma_{12}\right|$ is taken to be minimal, and if $\gamma_{12}$ and $\gamma_{34}$ are then coprime, it follows that $\left\{c_{12}, c_{34}\right\}$ is cyclic; for we can find integers $\delta$ and $\varepsilon$ for which

and we have

$$
\delta \gamma_{12}+\varepsilon \gamma_{34}=1
$$

$$
\left\{c_{12}, c_{34}\right\}=\left\{c_{12}^{e} c_{34}^{\delta}\right\} .
$$

Therefore we shall assume that $\left\{c_{12}, c_{43}\right\}$ is infinite but not torsion-free, and it will be convenient to assume that its periodic subgroup is a $p$-group for some prime $p$. For the elements in $G^{\prime}$ of finite order prime to any fixed $p$ form a characteristic subgroup of $G^{\prime}$ and so a normal subgroup $N$ of $G$; we may replace $G$ by $G / N$ without disturbing any of our assumptions about $c_{12}$ and $c_{34}$. What we shall show is that $\left\{c_{12}, c_{34}\right\}$ contains in fact no element of order $p$, and so is torsion-free. 
Consider the case in which $c_{12}$ is central in $G$. Then $\lambda_{k}=\mu_{k}=1$ for $1 \leqq k \leqq 4$; so $c_{34}$ is central in $G$. This ensures that the subgroup $M$ generated by the $m$ th powers of the elements of $\left\{c_{12}, c_{34}\right\}$ is normal, $m$ being the order of the periodic subgroup of $\left\{c_{12}, c_{34}\right\}$. Thus $c_{12} M$ and $c_{34} M$ have finite orders exceeding 1 ; so the subgroup $\left\{c_{12} M, c_{34} M\right\}$ of $G / M$ is cyclic, and its order is at most $m$. Therefore $m=1$, and then $\left\{c_{12}, c_{34}\right\}$ is cyclic, as we wished to prove.

For the rest of the proof of Theorem 3 we assume that neither $c_{12}$ nor $c_{34}$ is central in $G$, that is that, in the notation introduced above, some $\mu_{k}-\lambda_{k}$ is non-zero. We have that $\left[c_{12}, a_{k}\right]^{\lambda_{k}}$ belongs to $K\left(c_{12}\right)$ and is equal to

$$
d_{12}^{\left(-\lambda_{k}+\mu_{k}\right) \lambda_{k}}=c_{12}^{\mu_{k}-\lambda_{k}}
$$

for a suitable element $d_{12}$; this shows that $c_{12}$ has finite order modulo $K\left(c_{12}\right)$. Consequently, the relation (*) shows that $c_{34}$ has finite order modulo $K\left(c_{12}\right)$. A similar argument shows that $c_{12}$ and $c_{34}$ have finite orders modulo $K\left(c_{34}\right)$. If we put $K=K\left(c_{12}\right) \cap K\left(c_{34}\right)$, we see that $c_{12} K$ and $c_{34} K$ are elements of finite order in $G / K$. where

An earlier result now indicates that $\left\{c_{12} K, c_{34} K\right\}$ is a cyclic subgroup of $G / K$, say $\{c K\}$,

$$
c_{12} K=(c K)^{\alpha}, \quad c_{34} K=(c K)^{\beta},
$$

and $\alpha$ and $\beta$ are coprime. The relations

$$
\left(c_{12} K\right)^{\beta}=\left(c_{34} K\right)^{\alpha}, \quad c_{12}^{\beta} c_{34}^{-\alpha} \in K
$$

follow, and here we assume without losing generality that $\alpha$ and $p$ are coprime. Then we have

$$
c_{12}^{\beta} c_{34}^{-\alpha} \in K\left(c_{12}\right) \text {. }
$$

Next we examine a typical generator of $K\left(c_{12}\right)$. This has the form $\left[c_{12}, x\right]$, or (because $G^{\prime}$ is abelian) $\left[c_{12}, a\right]$, where $a=a_{1}^{n_{1}} a_{2}^{n_{2}} a_{3}^{n_{3}} a_{4}^{n_{4}}$ for certain integers $n_{k}$. If we suppose for the moment that each $n_{k}$ is positive, then there is an element $d$ such that

$$
c_{12}=d^{\lambda}, \quad \lambda=\lambda_{1}^{n_{1}} \lambda_{2}^{n_{2}} \lambda_{3}^{n_{3}} \lambda_{4}^{n_{4}} ; \quad c_{12}^{a}=d^{\mu}, \quad \mu=\mu_{1}^{n_{1}} \mu_{2}^{n_{2}} \mu_{3}^{n_{3}} \mu_{4}^{n_{4}} .
$$

It follows from this that

$$
\left[c_{12}, x\right]=\left[c_{12}, a\right]=d^{\mu-\lambda}, \quad\left[c_{12}, x\right]^{\lambda}=c_{12}{ }^{\mu-\lambda} .
$$

Relations of the same sort can be found when some $n_{k}$ are negative. For instance, when $n_{1}$ is negative and the rest are positive the last relation holds provided we take $\lambda$ to be $\mu_{1}^{n_{1}} \lambda_{2}^{n_{2}} \lambda_{3}^{n_{3}} \lambda_{4}^{n_{4}}$, and $\mu$ to be $\lambda_{1}^{n_{1}} \mu_{2}^{n_{2}} \mu_{3}^{n_{3}} \mu_{4}^{n_{4}}$.

We note that no $\lambda_{k}$ or $\mu_{k}$ is divisible by $p$. For we may assume that $\left\{c_{12}, c_{34}\right\}$ contains an element $c$ of order $p$, and for this element we have

$$
\left(c^{\lambda_{k}}\right)^{a_{k}}=c^{\mu_{k}}
$$

where $\lambda_{k}$ and $\mu_{k}$ are coprime. If $\mu_{k}$, for instance, was divisible by $p$, then the transformation of $G$ by $a_{k}$ would not be an automorphism. Therefore $\left[c_{12}, x\right]^{\lambda}$ lies in $\left\{c_{12}\right\}$, where $\lambda$ and $p$ are coprime. This with the earlier relation $c_{12}^{\beta} c_{34}^{-\alpha} \in K\left(c_{12}\right)$ shows that

$$
c_{12}^{\beta \omega} c_{34}^{-\alpha \omega} \in\left\{c_{12}\right\}
$$


for some number $\omega$ prime to $p$, and so

$$
c_{34}^{\omega_{1}}=c_{12}^{\omega_{2}}
$$

where $\omega_{1}$ is prime to $p$.

But the relation of this form with $\left|\omega_{1}\right|$ minimal, and the fact that elements of finite order in $\left\{c_{12}, c_{34}\right\}$ have $p$-power order, show (as explained earlier) that $\left\{c_{12}, c_{34}\right\}$ is cyclic.

This completes the proof of Theorem 3 .

COROLlaRY. The commutator subgroup of the group $G$ is locally cyclic if and only if $[g, x]$ and $[g, y]$ generate a cyclic subgroup, where $g, x$ and $y$ are arbitrary elements of $G$.

Proof. The lemma shows that our hypothesis implies that every $K(g)$ is locally cyclic. Application of Theorem 3 completes the proof.

Finally we describe a group $G_{3}$ in which $G_{3}^{\prime}$ is an arbitrary locally cyclic group while every $K(g)$ is cyclic. Let $F$ be the free nilpotent group of class two on two generators, take a countable infinity of copies of $F$, and let $P$ be the restricted directed product of all these groups. Thus the centre of $P$, which is also $P^{\prime}$, is a free abelian group of countably infinite rank. Now to any given locally cyclic group $L$ there corresponds a subgroup $N$ of $P$ such that $L$ is isomorphic to $P^{\prime} \mid N$; the example $G_{3}$ is defined to be $P / N$. The proof of the fact asserted about $K(g)$ is easy, and is omitted.

In particular a non-cyclic group, for instance the additive rationals, may be taken for $L$. This shows that Theorem 2 does not always hold when $G^{\prime}$ is infinite.

\section{REFERENCES}

1. I. D. Macdonald, On certain varieties of groups, Math. $Z$. 76 (1961), 270-282.

2. B. H. Neumann, Groups with finite classes of conjugate elements, Proc. London Math. Soc. (3) 1 (1951), 178-187.

3. B. H. Neumann, Groups covered by permutable subsets, J. London Math. Soc. 29 (1954), 236-248.

4. O. Ore, Some remarks on commutators, Proc. Amer. Math. Soc. 2 (1951), 307-314.

5. H. J. Zassenhaus, The theory of groups (New York, 1958).

THE UNIVERSITY

SHEFFIELD 10 